\title{
First Clinical Experience with Integrated Whole-Body PET/MR: Comparison to PET/CT in Patients with Oncologic Diagnoses
}

\author{
Alexander Drzezga*1, Michael Souvatzoglou*1, Matthias Eiber ${ }^{1,2}$, Ambros J. Beer ${ }^{1}$, Sebastian Fürst ${ }^{1}$, \\ Axel Martinez-Möller ${ }^{1}$, Stephan G. Nekolla ${ }^{1}$, Sibylle Ziegler ${ }^{1}$, Carl Ganter ${ }^{2}$, Ernst J. Rummeny ${ }^{2}$, \\ and Markus Schwaiger ${ }^{1}$ \\ ${ }^{I}$ Department of Nuclear Medicine, Technische Universität München, Munich, Germany; and ${ }^{2}$ Department of Radiology, Technische \\ Universität München, Munich, Germany
}

The recently introduced first integrated whole-body PET/MR scanner allows simultaneous acquisition of PET and MRI data in humans and, thus, may offer new opportunities, particularly regarding diagnostics in oncology. This scanner features major technologic differences from conventional PET/CT devices, including the replacement of photomultipliers with avalanche photodiodes and the need for MRI-based attenuation correction. The aim of this study was to evaluate the comparability of clinical performance between conventional $\mathrm{PET} / \mathrm{CT}$ and PET/MR in patients with oncologic diseases. Methods: Thirty-two patients with different oncologic diagnoses underwent a single-injection, dual-imaging protocol consisting of a PET/CT and subsequent PET/MR scan. PET/CT scans were performed according to standard clinical protocols ( $86 \pm 8$ min after injection of $401 \pm 42 \mathrm{MBq}$ of ${ }^{18} \mathrm{~F}-\mathrm{FDG}, 2 \mathrm{~min} /$ bed position). Subsequently (140 \pm 24 min after injection), PET/ MR was performed (4 min/bed position). PET images of both modalities were reconstructed iteratively. Attenuation and scatter correction as well as regional allocation of PET findings were performed using low-dose CT data for PET/CT and Dixon MRI sequences for PET/MR. PET/MR and PET/CT were compared visually by 2 teams of observers by rating the number and location of lesions suspicious for malignancy, as well as image quality and alignment. For quantitative comparison, standardized uptake values (SUVS) of the detected lesions and of different tissue types were assessed. Results: Simultaneous PET/MR acquisition was feasible with high quality in short acquisition time ( $\leq 20 \mathrm{~min}$ ). No significant difference was found between the numbers of suspicious lesions $(n=80)$ or lesion-positive patients $(n=20)$ detected with PET/MR or PET/CT. Anatomic allocation of PET/ MR findings by means of the Dixon MRI sequence was comparable to allocation of PET/CT findings by means of low-dose CT. Quantitative evaluation revealed a high correlation between mean SUVs measured with PET/MR and PET/CT in lesions $(\rho=0.93)$ and background tissue $(\rho=0.92)$. Conclusion: This study demonstrates, for what is to our knowledge the first time, that integrated whole-body PET/MR is feasible in a clinical setting with

Received Sep. 26, 2011; revision accepted Jan. 10, 2012.

For correspondence or reprints contact: Alexander Drzezga, Department of Nuclear Medicine, Technische Universität München, Klinikum rechts der Isar, Ismaninger Strasse 22, 81675 Munich, Germany.

E-mail: a.drzezga@Irz.tum.de

Published online Apr. 25, 2012.

${ }^{*}$ Contributed equally to this work.

COPYRIGHT @ 2012 by the Society of Nuclear Medicine, Inc. high quality and in a short examination time. The reliability of PET/ MR was comparable to that of PET/CT in allowing the detection of hypermetabolic lesions suspicious for malignancy in patients with oncologic diagnoses. Despite different attenuation correction approaches, tracer uptake in lesions and background correlated well between PET/MR and PET/CT. The Dixon MRI sequences acquired for attenuation correction were found useful for anatomic allocation of PET findings obtained by PET/MR in the entire body. These encouraging results may form the foundation for future studies aiming to define the added value of PET/MR over PET/CT.

Key Words: hybrid imaging; PET/MR; PET/CT; multimodal imaging; oncology

J Nucl Med 2012; 53:845-855

DOI: 10.2967/jnumed.111.098608

$\mathbf{R}$ ecently, the first whole-body integrated PET/MR scanner (Biograph mMR; Siemens Healthcare) was introduced. This new technology bears the potential to repeat the success of PET/CT, particularly for oncologic indications, which are better addressed with MRI than with CT. With regard to soft-tissue contrast, CT is known to be clearly inferior to MRI. This is the reason why MRI is regarded as the first-line imaging procedure for diagnostic problems in oncology associated with soft-tissue regions (e.g., tumors in the brain, the head-and-neck region, or the pelvis). Because PET has proven valuable for several of these diagnostic problems, a potential added value of combined MRI and PET over PET/CT can be expected for these indications (1-5). In principle, PET and MRI data may be fused in retrospect using dedicated software registration algorithms; however, these approaches often encounter problems with patient position and motion $(6,7)$.

Consequently, first platforms have been developed in which spatially separated MRI and PET scanners are positioned side by side and connected by means of a moving patient table on which the patient undergoes PET and MRI consecutively (8). However, these solutions do not allow 
simultaneous image acquisition, are associated with lengthy examination protocols, and still bear the risk of misalignment due to patient movement. The replacement of conventional photomultipliers with avalanche photodiodes, which are not affected by strong magnetic fields, allowed the integration of PET and MRI technology in a single machine $(9,10)$. The feasibility of this concept has been demonstrated in small-animal scanners and in a first generation of head-only human PET inserts that could be flexibly installed inside the bore of conventional MRI tomographs (11-14).

A Biograph mMR was installed in November 2010 in the Department of Nuclear Medicine at the Technische Universität München, funded by the German Research Foundation (Deutsche Forschungsgemeinschaft). The Biograph mMR consists of a high-end 3-T MRI scanner (technically corresponding to the Siemens Verio system) that harbors a fully functional state-of-the-art avalanche photodiodebased PET system within its gantry. The scanner is operated by a consortium between the Departments of Nuclear Medicine and Radiology from Technische Universität München and from the Ludwig-Maximilians-Universität.

This scanner features several basic technical differences from a conventional PET/CT scanner. Two factors may affect the quality of the acquired PET data: the replacement of the photomultipliers with avalanche photodiodes, and the attenuation correction of PET data acquired in the PET/MR scanner, which has to be derived from MRI scan information. In conventional PET/CT scanners, attenuation correction is performed on the basis of a CT scan that is used to generate an attenuation map based on a transformation of the CT Hounsfield units into attenuation factors at $511 \mathrm{keV}$ (15). Naturally, the MRI signal does not provide information on the radiodensity of the tissue and cannot directly be used for attenuation correction. Several alternative approaches have been discussed, including anatomically based attenuation maps and automatic atlas-based pattern recognition approaches (16-20). The procedure implemented in the Biograph mMR uses an MRI-based attenuation map that is generated on the basis of a 2-point Dixon MRI sequence (19). The Dixon sequence allows estimation of the distribution of 4 different tissue types (fat, soft tissue, lungs, and background/air) throughout the body and the calculation of an attenuation map on the basis of the presumed radiodensity of these tissue types $(19,21,22)$. Although disregarding attenuation by cortical bone, this technique has been suggested to show performance comparable to that of CT-based attenuation $(19,23)$. However, so far the quality of clinical PET data acquired on an integrated whole-body PET/MR scanner and attenuation-corrected by means of MRI data has not been systematically compared with PET data acquired on a PET/CT scanner in the same patients.

Another difference between PET/MR and PET/CT is that the anatomic allocation of PET findings in the whole body has to be performed by means of the MRI data rather than by the information provided by CT. The PET signal alone provides only limited anatomic information. In conventional PET/CT, low-dose CT information is used not only for attenuation correction but also for anatomic allocation of PET findings, when diagnostic CT is not performed (24). In addition, even on low-dose CT some PET-negative pathologies can be detected, such as small pulmonary lesions or bone metastases $(25,26)$. MRI offers high-resolution diagnostic sequences, providing detailed anatomic information. However, because of relatively long acquisition times, usually high-resolution sequences are acquired only in selected regions of interest and in 1 preferred anatomic orientation (nonisotropic). Also for PET/MR, time constraints may not allow covering the whole body with fully diagnostic MRI sequences, and predominantly nonisotropic data will be acquired. However, unpredicted PET findings may also be detected in regions not covered by high-resolution MRI, and anatomic information may also be necessary to distinguish physiologic tracer uptake from pathologic findings throughout the body. Thus, a fast method for rough anatomic allocation of PET findings may be valuable also for regions in which diagnostic sequences have not been performed. In this context, a recently published paper from Eiber et al. concluded that images derived from the 2-point Dixon MRI sequence, which is performed for attenuation correction in a short time (19 s acquisition time) for each PET bed position (BP), may have considerable value for the anatomic allocation of PET findings throughout the body (23).

The aim of this study was to evaluate the clinical performance of integrated whole-body PET/MR as compared with PET/CT in 2 aspects: First, is the quality of the PET data obtained with PET/MR comparable to that obtained with PET/CT, regarding the detectability of lesions suspicious for malignancy in patients with oncologic diagnoses, despite different PET detector technologies and attenuation correction procedures? Second, will anatomic allocation of the suggestive PET findings in PET/MR (by means of the MRI Dixon images) in the entire body be as reliable as that of low-dose CT in conventional PET/CT?

\section{MATERIALS AND METHODS}

\section{Patient Population}

Of the pool of patients routinely referred to our institute for clinical ${ }^{18} \mathrm{~F}$-FDG PET/CT for staging and follow-up of malignant disorders, 32 subjects were recruited consecutively for this prospective study (mean age, $61 \pm 11 \mathrm{y}, 21$ male, 11 female, Table 1). Oncologic diagnoses included anal cancer, breast cancer, colon cancer, carcinoma of unknown primary, esophageal cancer, sarcoma, leukemia, lymphoma, and pancreatic cancer (Table 1).

After their clinical PET/CT scan, the patients underwent a PET/ MR examination on the integrated whole-body scanner. All patients gave informed consent, and the approval of the institutional review board and the radiation protection authorities had been obtained. The inclusion criteria were informed consent, ability to undergo another scan after the PET/CT examination, and a clinical indication for ${ }^{18} \mathrm{~F}$ FDG PET with a low-dose CT scan. Exclusion criteria were pregnancy, age below $18 \mathrm{y}$, and standard contraindications for MRI 
TABLE 1

Patient Characteristics

\begin{tabular}{|c|c|c|c|c|c|}
\hline Patient no. & Sex & Age & Type of malignancy & No. of suggestive PET-positive findings & No. of lesions \\
\hline 1 & $\mathrm{M}$ & 54 & Anal cancer & Lymph nodes (3), primary tumor (1) & 4 \\
\hline 2 & $\mathrm{~F}$ & 69 & Bronchial carcinoma & Lung (1) & 1 \\
\hline 3 & $\mathrm{~F}$ & 44 & Breast cancer & Bone (5) & 5 \\
\hline 4 & $\mathrm{~F}$ & 67 & Breast cancer & Breast (1) & 1 \\
\hline 5 & $\mathrm{~F}$ & 69 & Breast cancer & Bone (5), lymph nodes (2). liver (1) & 8 \\
\hline 6 & $\mathrm{M}$ & 60 & Colon cancer & Liver (5) & 5 \\
\hline 7 & $\mathrm{~F}$ & 68 & Colon cancer & Liver (1) & 1 \\
\hline 8 & $\mathrm{~F}$ & 73 & Colon cancer & Lymph nodes (3) & 3 \\
\hline 9 & $\mathrm{M}$ & 44 & Colon cancer & Liver (1) & 1 \\
\hline 10 & $\mathrm{M}$ & 61 & Carcinoma of unknown primary & Lymph nodes (2) & 2 \\
\hline 11 & $\mathrm{M}$ & 70 & Carcinoma of unknown primary & No suggestive findings & 0 \\
\hline 12 & $\mathrm{M}$ & 54 & Carcinoma of unknown primary & No suggestive findings & 0 \\
\hline 13 & $\mathrm{M}$ & 61 & Carcinoma of unknown primary & Prostate (1) & 1 \\
\hline 14 & $\mathrm{M}$ & 49 & Esophageal cancer & No suggestive findings & 0 \\
\hline 15 & $\mathrm{~F}$ & 25 & Ewing sarcoma & Lung (2), bone (3), peritoneum (2), pleura (1) & 8 \\
\hline 16 & $\mathrm{~F}$ & 68 & Hepatocellular carcinoma & Liver (1) & 1 \\
\hline 17 & $\mathrm{M}$ & 66 & Hepatocellular carcinoma & No suggestive findings & 0 \\
\hline 18 & $\mathrm{M}$ & 71 & Hepatocellular carcinoma & Adrenal gland (1), liver $\left(2^{\star}\right)$ & 3 \\
\hline 19 & $\mathrm{M}$ & 52 & Leukemia & No suggestive findings & 0 \\
\hline 20 & $\mathrm{M}$ & 51 & Leukemia & No suggestive findings & 0 \\
\hline 21 & $\mathrm{M}$ & 72 & Lymphoma & Lymph nodes (3), lung (1) & 4 \\
\hline 22 & $\mathrm{M}$ & 67 & Lymphoma & Lymph nodes $\left(9^{\dagger}\right)$, bone (1), spleen(1), skin (1) & 12 \\
\hline 23 & $\mathrm{M}$ & 71 & Melanoma & No suggestive findings & 0 \\
\hline 24 & $\mathrm{~F}$ & 71 & Melanoma & No suggestive findings & 0 \\
\hline 25 & $\mathrm{M}$ & 62 & Melanoma & No suggestive findings & 0 \\
\hline 26 & $\mathrm{~F}$ & 53 & Melanoma & Lung (1), lymph nodes (1) & 2 \\
\hline 27 & $\mathrm{M}$ & 48 & Melanoma & No suggestive findings & 0 \\
\hline 28 & $\mathrm{M}$ & 55 & Melanoma & No suggestive findings & 0 \\
\hline 29 & $\mathrm{M}$ & 65 & Pancreatic cancer & No suggestive findings & 0 \\
\hline 30 & $\mathrm{M}$ & 65 & Rectal cancer & Lymph nodes (2) & 2 \\
\hline 31 & $\mathrm{~F}$ & 64 & Rectal cancer & Lung (5), rectum (1) & 6 \\
\hline 32 & $\mathrm{M}$ & 81 & Thyroid cancer & Lung (5), pleura (1), lymph nodes(4) & 10 \\
\hline
\end{tabular}

*Detected only on PET/CT.

${ }^{\dagger}$ Detected in different compartments.

examinations (magnetic metal implants, pacemakers, etc.). We did also not include patients undergoing diagnostic CT with application of intravenous contrast material, to ensure homogeneity in the datasets used for CT-based attenuation correction.

\section{Imaging Protocol}

All subjects underwent a single-injection, dual-imaging protocol including PET/CT and subsequent PET/MR. After completion of the PET/CT scan, patients were subsequently positioned on the PET/MR scanner with the smallest possible temporal delay, to allow for using the remaining activity of the initial ${ }^{18}$ F-FDG injection. Consequently, this approach did not require additional injection of ${ }^{18} \mathrm{~F}-\mathrm{FDG}$ and, thus, was not associated with any additional radiation exposure for the patients.

\section{PET/CT Acquisition}

PET/CT acquisition was performed according to standard clinical protocols, as previously reported (23), on a Biograph Sensation $64 \mathrm{PET} / \mathrm{CT}$ scanner (Siemens Medical Solutions). The scanner has an average spatial resolution of $4.4 \mathrm{~mm}$ at $1 \mathrm{~cm}$ and of $5.0 \mathrm{~mm}$ at $10 \mathrm{~cm}$ from the transverse field of view (FOV) and a maximum sensitivity of $8.1 \mathrm{kcps} / \mathrm{MBq}$ at the center of the FOV. Its axial FOV is $21.8 \mathrm{~cm}$ (27). Patients fasted for at least $6 \mathrm{~h}$ before undergoing scanning, and blood glucose levels were measured just before injection to ensure a value below $150 \mathrm{mg} / \mathrm{dL}$. Patients were injected with $401 \pm 42 \mathrm{MBq}$ of ${ }^{18} \mathrm{~F}$-FDG intravenously depending on their body weight, and the acquisition was started $86 \pm 8 \mathrm{~min}$ after injection, moving from pelvis to head. The acquisition time was 2 min per BP, with 5-6 BPs (each $21 \mathrm{~cm}$ ) covering the trunk of the patients. The result was a total acquisition time of approximately $15 \mathrm{~min}$ per patient for PET/CT. PET BPs started from the pelvis and moved up toward the head. For attenuation correction, low-dose CT (120 keV, $20 \mathrm{mAs})$ in shallow inspiration was performed. Patients received an oral contrast agent (ioxithalamate [Telebrix]; Guerbet, $15 \mathrm{~mL}$ diluted in $1 \mathrm{~L}$ of water), but no intravenous contrast agent was applied. For both modalities, a head pillow and knee cushion were used for positioning the patients, to render positioning comparable in both scanners.

\section{PET/MR}

Instrumentation. PET/MR was performed on the Biograph $\mathrm{mMR}$. The technical specifications were summarized recently in a performance evaluation paper (28). In brief, this system consists of a 3-T MRI scanner featuring high-performance gradient systems (45 $\mathrm{mT} / \mathrm{m}$ ) and a slew rate of $200 \mathrm{~T} / \mathrm{m} / \mathrm{s}$. The PET/MR system is equipped with Total Imaging Matrix coil technology (Siemens), 
covering the entire body with multiple integrated radiofrequency surface coils. This technology allows MRI acquisitions of the whole body without the need to interrupt the examination for repositioning of coils for different body regions (29). The coils (and all other equipment such as patient table and cables) have been redesigned for PET/MR in order to minimize their attenuation and, thus, to allow unimpaired PET acquisition with the coils in place. Within the gantry, the MRI scanner harbors a fully functional PET system, equipped with the avalanche photodiode technology (9). The PET scanner has a spatial resolution of 4.3 at $1 \mathrm{~cm}$ and of $5.0 \mathrm{~mm}$ at $10 \mathrm{~cm}$ from the transverse FOV and a sensitivity of $15.0 \mathrm{kcps} / \mathrm{MBq}$ at the center of the FOV. The axial FOV is $25.8 \mathrm{~cm}$ (28), which allows covering the entire body with a low number of BPs in a short time. For calibration, during the daily quality assurance procedure, a cylindric ${ }^{68} \mathrm{Ge}$ phantom is centered in the FOV and the ECAT count conversion factors and normalization files are produced, according to the National Electrical Manufacturers Association criteria.

Imaging Protocol. On average, the PET/MR scan was started $140 \pm 24$ min after injection. Patients were positioned in the MRI scanner as similarly as possible to their positioning for the PET/ CT examination. Acquisition started in the pelvic region and moved toward the head. First, a localizer MRI scan was performed to define the BPs. After correct positioning of the spatial acquisition windows had been ensured, the combined PET/MR acquisition was initiated with 3-5 BPs at a 4-min acquisition time per BP. First, a coronal 2-point Dixon 3-dimensional volumetric interpolated breath-hold T1-weighted MRI sequence was acquired at each BP and used for the generation of attenuation maps and for anatomic allocation of the PET results, as previously described $(19,23)$. The parameters for this sequence were as follows: integrated parallel acquisition technique; factor, 2; voxel size, $4.1 \times$ $2.6 \times 3.1 \mathrm{~mm}$ (in-plane resolution $\times$ slice thickness); acquisition time, $19 \mathrm{~s}$; repetition time, $3.6 \mathrm{~ms}$, first echo time, $1.225 \mathrm{~ms}$; second echo time, $2.45 \mathrm{~ms}$; matrix, $79 \times 192$; number of excitations, 1; FOV, 500 mm; phase FOV, 65.5\%; 1 slab with 128 slices; slice thickness, $3.1 \mathrm{~mm}$; flip angle, $10^{\circ}$; and bandwidth, $960 \mathrm{~Hz} /$ pixel. To minimize artifacts from an incomplete breath-hold, a centric k-space acquisition was chosen (30). No contrast agent was administered for the MRI studies. The software of the MRI scanner automatically used the raw images to generate 4 different images: T1-weighted in-phase, T1-weighted out-of-phase, water-only, and fat-only.

Simultaneously with the start of the Dixon MRI sequence, the PET acquisition started at the same BP, thus ensuring optimal temporal and regional correspondence between MRI and PET data. The PET acquisition time was 4 min per BP, taking delayed acquisition times and radioactive decay into account. After completion of the PET acquisition, the table was moved to the next BP and the procedure was repeated. In the thorax and abdomen regions, the MRI scans were acquired during breath-hold in shallow inspiration, similarly to the acquisition of the low-dose CT. Subsequently, the patients were instructed to continue breathing during the remaining PET acquisition time. Because of the large FOV $(25.8 \mathrm{~cm})$, the trunk of the patients could be covered within 3-5 BP, resulting in an average total examination time below $20 \mathrm{~min}$.

\section{Data Processing}

Reconstruction. PET data obtained on the PET/CT and PET/MR scanners were processed with comparable reconstruction and correction algorithms. For both modalities, emission data were corrected for randoms, dead time, scatter, and attenuation. A 3-dimensional attenu- ation-weighted ordered-subsets expectation maximization iterative reconstruction algorithm (AW OSEM 3D) was applied with 3 iterations and 21 subsets, gaussian smoothing of $4 \mathrm{~mm}$ in full width at half maximum, and a zoom of 1 . Attenuation maps were obtained from the CT data by bilinear transformation, as implemented in the postprocessing software of the PET/CT scanner, and were used for attenuation correction of the PET/CT data, as previously described (15).

MRI-Based Attenuation Correction. For attenuation correction of the PET data from the PET/MR scanner, attenuation maps generated on the basis of the Dixon MRI sequence were applied, as recently published $(19,23)$. The attenuation maps were generated on the basis of the 2-point Dixon MRI sequences obtained for every BP. This approach has recently been demonstrated to provide results comparable to those of conventional attenuation correction by lowdose CT (19,23). The procedure has been implemented in the postprocessing software of the scanner and operates automatically. The Dixon fat- and water-weighted images were used to create an attenuation map with 4 distinct tissue-classes: background, lungs, fat, and soft tissue. The lungs were identified by connected-component analysis of the air in the inner part of the body. By application of a morphologic closing filter, virtual air artifacts induced by the absence of an MRI signal in cortical bone, heart, and aorta (because of blood flow) were corrected. Attenuation of the PET signal caused by instrumentation such as the patient bed and the fixed MRI coils is automatically integrated into the attenuation maps (31). The flexible Total Imaging Matrix coils have been specially designed for low attenuation and are not included in the attenuation maps.

\section{Image Analysis}

Visual Rating. Images were analyzed by 4 experienced (boardcertified) readers (2 nuclear medicine physicians and 2 radiologists). The readers were grouped into 2 teams, each containing 1 nuclear medicine physician and 1 radiologist. The acquired scans were distributed to the 2 groups in the following fashion: Of 32 patients who had undergone PET/CT and PET/MR, the PET/CT data of patients $1-16$ and the PET/MR data of patients 17-32 were read by team 1, and the PET/MR data of patients 1-16 and PET/ CT data of patients $17-32$ were read by team 2 . Thus, readers would never have to rate the PET/CT and PET/MR scans of the same patient. Using this approach, we aimed to avoid potential bias due to the circumstance that readers would potentially detect a lesion more easily in one modality after knowing it from the other modality. To minimize interobserver variability, we required that concordant conclusions regarding the number, location, and dignity (i.e. benign vs. malignant) of PET findings had to be drawn within teams. The entire rating procedure was performed on a dedicated workstation and software (Syngo MMWP and Syngo TrueD; Siemens Medical Solutions). For rating of the PET/CT data, PET images were screened for any suggestive focal uptake, and the coregistered low-dose CT scan was used for anatomic correlation and for identification of a potential structural correlate in the CT scan. Similarly, for rating of the PET/MR data, PET images were used to identify any region with suggestive focal uptake, and all 4 coregistered Dixon MRI datasets (T1-weighted in-phase, T1weighted out-of-phase, fat-only, and water-only) were evaluated for anatomic allocation of the finding and for the presence of a distinct lesion corresponding to the focal uptake.

The ${ }^{18} \mathrm{~F}$-FDG PET data from both modalities were rated globally for every patient with regard to the presence, number, and location of findings suggestive of malignancy. In analogy to a recent publication (23), a scoring scale ranging between 0 and 3 was used for 
visual ratings of 4 features. First, the subjective contrast of the detected lesion in the PET image was rated by judging the visual detectability of the lesion versus the respective surrounding background tissue ( 0 , not detectable; 1 , low contrast; 2 , intermediate contrast; 3 , high contrast). The second rating was of the feasibility of anatomic allocation of the PET finding using the low-dose CT scan and the MRI Dixon sequences ( 0 , no anatomic correlation possible/no morphologic correlate detectable; 1 , uncertain anatomic correlation/no morphologic correlate detectable; 2, good anatomic correlation/questionable morphologic correlate; and 3, excellent anatomic correlation with a clear morphologic correlate). Third, the overall image quality of the PET data ( 0 [bad] to 3 [very good]) was rated on the basis of the subjective impression of the overall quality of the PET data (including, for example, impressions of smoothness/scatteredness, resolution, sharpness of contours, anatomic detail, homogeneity, and presence of artifacts). Fourth, the quality of the alignment between PET and structural image data was rated $(0$, major misalignment of several organ systems; 1 , major misalignment of the dome of the liver/diaphragm; 2, mild misalignment of the dome of the liver/diaphragm; 3 , no apparent misalignment).

As in previous studies, in the case of excessive numbers of PET-positive lesions in a single organ system or compartment, up to 5 lesions only per organ system or compartment were chosen to avoid bias from individual patients (23). We decided to select the first 5 lesions in order of appearance when screening the patient data on axial slices in the craniocaudal direction. Using this approach, we tried to avoid bias induced by the subjective selection of lesions based on their contrast or size. To ensure that in these cases the identical lesions had been rated by the observing teams in the PET/MR and PET/CT datasets, we performed a retrospective comparison with both teams present.

Quantitative Assessment. For quantitative comparison between the PET data acquired on the PET/CT and PET/MR scanners, we performed a standarized uptake value (SUV)-based analysis of tracer uptake in the suspected lesions and in different normal organ systems (lung, vertebral bone, liver, spleen, and muscle). Volumes of interest (VOIs) were placed over matching ${ }^{18} \mathrm{~F}-\mathrm{FDG}$ images of suspected tumor lesions and organs. To ensure placement of VOIs in corresponding locations, both PET scans were coregistered by dedicated software (TrueD; Siemens). The correctness of coregistration was controlled visually. To calculate SUVs for suspected tumor lesions, the axial slice with the maxi- mum SUV of the lesion was first located automatically, using standardized software, for the images of both scanners. An isocontour VOI including all voxels above $50 \%$ of the maximum was then created to calculate mean SUVs. This method was selected on the basis of a previous study suggesting that this approach has a high accuracy (32). To avoid overrating of individual patients, we limited the number of rated lesions to 5 per patient. For the 2 modalities, the SUVs of the organs were determined by placement of regions of interest in 3 consecutive anatomically corresponding central axial slices through the organ in question, delineating the shape of the organ but not including edge pixels. The mean of all organ measurements was used to define a background SUV for every patient. Within all VOIs, mean and maximum SUVs were measured. The volume of the automatically defined VOIs was assessed as well and was compared between the 2 modalities.

\section{Statistical Analysis}

To test agreement on the number of detected lesions between PET/CT and PET/MR, we used a Fisher exact test. The Kolmogorov-Smirnov test was used to test for a normal distribution. For calculating the overall statistical differences in measured SUVs and visual ratings between the 2 imaging modalities, we used a nonparametric Wilcoxon matched-pairs signed rank test for nonnormally distributed samples. The Spearman rank correlation coefficient $(\rho)$ was calculated to examine the correlation between the visual ratings and the mean and maximum SUVs derived from PET/MR and PET/CT. A $P$ value below 0.05 was considered significant.

\section{RESULTS}

\section{Number of Detected Lesions, Rating of Subjects}

Generally, PET results obtained by PET/MR showed good correspondence with PET results obtained by PET/CT (Fig. 1). In 20 of the 32 included subjects $(62.5 \%)$, suspected lesions were detected with both PET/MR and PET/ CT. All subjects who had been rated positive or negative for suspected lesions on PET/CT were also rated positive or negative on PET/MR. With PET/CT, a total of 80 suspected lesions were detected. Of these lesions, 78 were observed with PET/MR accordingly (i.e., in the same subjects at the

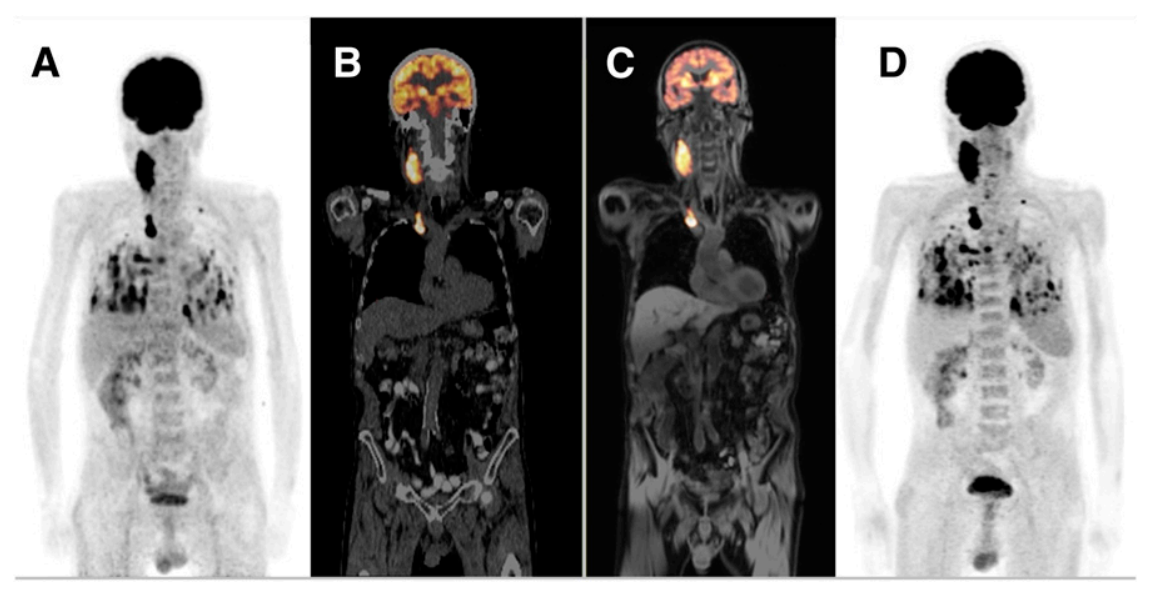

FIGURE 1. Proof of principle: comparison between PET/CT and PET/MR data acquired on same day in same patient with metastasized thyroid carcinoma. (A) ${ }^{18} \mathrm{~F}-\mathrm{FDG}$ PET acquired on PET/CT scanner displayed as maximum-intensity projection. (B) Overlay of PET (in orange) and structural low-dose CT data acquired on PET/CT scanner. (C) Overlay of PET (in orange) and water-weighted Dixon MRI acquired on PET/MR scanner. (D) ${ }^{18}$ F-FDG PET acquired on PET/MR scanner, displayed as maximum-intensity projection. An overall similarity can be seen in pattern of suspicious lesions in cervical, mediastinal, and pulmonary regions in A and D. 
identical locations). In only 1 patient, 2 liver lesions were clearly detectable in the PET/CT dataset but were missed by the team reading the PET/MR scans (patient 18). On retrospective evaluation by both teams, these lesions could also be identified in the PET/MR dataset but with low contrast. In 12 of the 32 subjects, no suspected lesions were detected with either of the 2 imaging modalities. In total, 29 lymph node metastases, 15 lung nodules, 14 bone lesions, 11 liver lesions, 2 pleural lesions, 2 peritoneal lesions, and 2 rectal lesions were observed, as well as single lesions in the spleen, prostate, breast, adrenal gland, and skin (Table 1). In patients with excessive numbers of lesions, only 5 lesions were counted per single organ system or compartment, to avoid bias by individuals with many lesions. The Fisher exact test did not reveal a significant difference $(P=0.5)$ between the number of lesions detected on PET/MR and the number detected on PET/CT (Table 2).

\section{Anatomic Allocation of PET-Positive Lesions}

According to the subjective rating, anatomic allocation of suspected PET-positive lesions with good reliability was possible both using low-dose CT (mean rating, 2.61 of 3) and using MRI Dixon images (mean rating, 2.03-2.42 of 3, depending on the type of sequence used, Table 3; Figs. 2 and 3). Numerically, the best performance was observed for anatomic allocation of PET findings by means of low-dose CT. However, no significant difference was observed between the scores obtained for the low-dose CT and the scores obtained with the opposed-phase and waterweighted Dixon MRI scans. The water-weighted images and opposed-phase images had similar ratings, without a significant difference. In contrast, anatomic allocation based on the in-phase or fat-weighted images was rated significantly worse than that based on low-dose CT and opposed-phase or water-weighted MRI scans.

The Spearman correlation analysis revealed generally low, nonsignificant correlation coefficients between the scores for the MRI sequences and those for low-dose CT (Table 3). Scores for the quality of anatomic allocation of the PET findings correlated strongly among all MRI sequences, with the highest correlation coefficient being between opposed-phase and in-phase images and the lowest being between the water- and the fat-weighted images.

\section{Image Quality, Contrast, Alignment}

The subjective rating of the contrast of suspected foci revealed no significant difference between values obtained for PET/MR and PET/CT (Fig. 1; Table 2). Alignment between PET scans and the anatomic scans was judged to be slightly better for PET/MR than for PET/CT, but this difference did not reach statistical significance. Subjective evaluation of general image quality revealed high ratings for both modalities, with a somewhat better result for PET/ CT. The observers reported moderately inhomogeneous liver uptake in 12 of the PET scans acquired on the PET/ MR scanner. Otherwise, no systematic artifacts were described by the observers for any of the modalities.

A significant correlation in the ratings of lesion contrast was found between PET/MR and PET/CT $(\rho=0.75)$. The ratings of image quality and alignment did not correlate significantly between PET/MR and PET/CT.

\section{SUV-Based Evaluation}

On the basis of the predefined assessment protocol, SUV analysis was performed for 63 lesions in all 20 lesion-positive patients and in different organ systems. In this analysis, a significant decrease was observed between the SUVs (mean and maximum) measured in the PET/CT data and in the subsequent PET/MR data. This was the case for the values obtained for suspected lesions, as well as for background and organ VOIs (liver, spleen, bone, muscle, and lung). For lesions, a mean difference between the SUV mean values of $9.4 \% \pm 18.4 \%$ (PET/CT $>$ PET/ MR) was observed, with a range between $-40.5 \%$ (PET/ $\mathrm{CT}<\mathrm{PET} / \mathrm{MR}$ ) and $49.7 \%$ (PET/CT $>$ PET/MR). For the background measurements, a mean reduction of $24.5 \% \pm$ 22.6\% (PET/CT > PET/MR) was found, with a range from $-31.6 \%$ (PET/CT < PET/MR) to $79 \%$ (PET/CT > PET/MR). (Table 4; Fig. 4). Because of the stronger decrease of background SUVs, we observed a higher quantitative lesion contrast in the PET/MR data than in the PET/CT data. This contrast was demonstrated by the semiquantitative evaluation of the lesion-to-muscle ratio, which increased significantly for the later-acquired PET/ MR data (Fig. 4). In the patient for whom the 2 liver lesions had been missed on first inspection of the PET/ MR scan, the SUV decreased strongly for the lesion and also for the background between PET/CT and PET/MR

TABLE 2

Visual Rating

\begin{tabular}{lccc}
\hline \multicolumn{1}{c}{ Parameter } & PET/CT & PET/MR & NS \\
\hline No. of lesion-positive patients (of 32) & 20 & 20 & NS \\
Total no. of detected lesions & 80 & 78 & NS \\
Subjective lesion-contrast PET (0-3), mean \pm SD & $2.76 \pm 0.56$ & $2.69 \pm 0.58$ & 0.02 \\
Subjective image quality (0-3), mean \pm SD & $2.93 \pm 0.26$ & $2.52 \pm 0.69$ & NS \\
Alignment (0-3), mean \pm SD & $2.72 \pm 0.60$ & $2.90 \pm 0.31$ &
\end{tabular}

Visual rating (0-3): $0=$ bad, $3=$ good.

$\mathrm{NS}=$ not statistically significant. 
TABLE 3

Anatomic Allocation

\begin{tabular}{|c|c|c|c|c|c|}
\hline Parameter & Low-dose CT & $\begin{array}{c}\text { Opposed-phase } \\
\text { MRI }\end{array}$ & $\begin{array}{c}\text { Water-weighted } \\
\text { MRI }\end{array}$ & $\begin{array}{l}\text { In-phase } \\
\text { MRI }\end{array}$ & $\begin{array}{c}\text { Fat-weighted } \\
\text { MRI }\end{array}$ \\
\hline $\begin{array}{l}\text { Rating of anatomic allocation (0-3), } \\
\text { mean } \pm S D\end{array}$ & $2.61 \pm 0.67$ & $2.42 \pm 0.87$ & $2.39 \pm 0.86$ & $2.24 \pm 0.92^{*}$ & $2.03 \pm 0.97^{\star \dagger}$ \\
\hline \multicolumn{6}{|l|}{ Spearman correlation coefficient $(\rho)$} \\
\hline Low dose CT & & NS & NS & NS & NS \\
\hline Opposed-phase MRI & & & 0.81 & 0.90 & 0.62 \\
\hline Water-weighted MRI & & & & 0.75 & 0.61 \\
\hline In-phase MRI & & & & & 0.72 \\
\hline $\begin{array}{l}{ }^{*} \text { Significantly lower scores as compa } \\
{ }^{\dagger} \text { Significantly lower scores as compa } \\
\text { Visual rating (0-3): } 0=\text { bad, } 3=\text { goo } \\
\text { NS = not statistically significant. }\end{array}$ & $\begin{array}{l}\text { with low-dose } \\
\text { with in-phase }\end{array}$ & $\begin{array}{l}\mathrm{T} \text {, opposed-phase } \\
\mathrm{RI}(P<0.05) .\end{array}$ & RI, and water-weic & ted MRI $(P<0$ & \\
\hline
\end{tabular}

(lesions, 39\%; background, 38\%). Consequently, the lesion-to-background contrast did not increase in this case, in contrast to other cases.

Correlation Analysis of Quantitative Values. Correlation analysis of the tracer uptake expressed by SUVs demonstrated a strong correlation in the mean SUVs of suspected lesions between PET/MR and PET/CT $(\rho=0.93)$ (Table 4; Fig. 5). Also, a high correlation was found between the PET/CT and PET/MR measurements of background SUVs (all organ VOIs combined) $(\rho=0.92)$. Lower correlations in the SUVs measured in individual organ systems were found between PET/CT and PET/MR, with the highest correlation detected for bone, intermediate values being found for liver and lung, and no significant correlation being found for muscle or spleen (Table 4).

Volumetric Assessment. Similarly to the maximum and mean SUVs, the mean volume of the automatically defined VOIs was significantly lower for PET/MR (mean, $6.2 \pm 8.2$ $\mathrm{cm}^{3}$ ) than for PET/CT (mean, $8.5 \pm 11.5 \mathrm{~cm}^{3}$ ), but the individual volumes correlated strongly between the 2 modalities $(\rho=0.97)$.

\section{DISCUSSION}

The results of this study indicate that integrated wholebody PET/MR imaging is feasible and produces PET information similar in quality to data acquired on a conventional PET/CT scanner. The effective increase in the axial FOV-nearly 31\%-for PET/MR allowed the same distance to be covered with fewer BPs, resulting in a short examination time $(<20 \mathrm{~min})$. This may be further reduced when shorter acquisition times per BP are applied. These encouraging results were obtained despite differences in the PET acquisition technologies (photomultipliers for PET/CT and avalanche photodiodes for PET/MR) and in the approaches to attenuation correction (CT-based for PET/ CT and MRI-based for PET/MR).

Importantly, the detectability of lesions with increased ${ }^{18} \mathrm{~F}-\mathrm{FDG}$ uptake (presumably representing ${ }^{18} \mathrm{~F}-\mathrm{FDG}$-avid

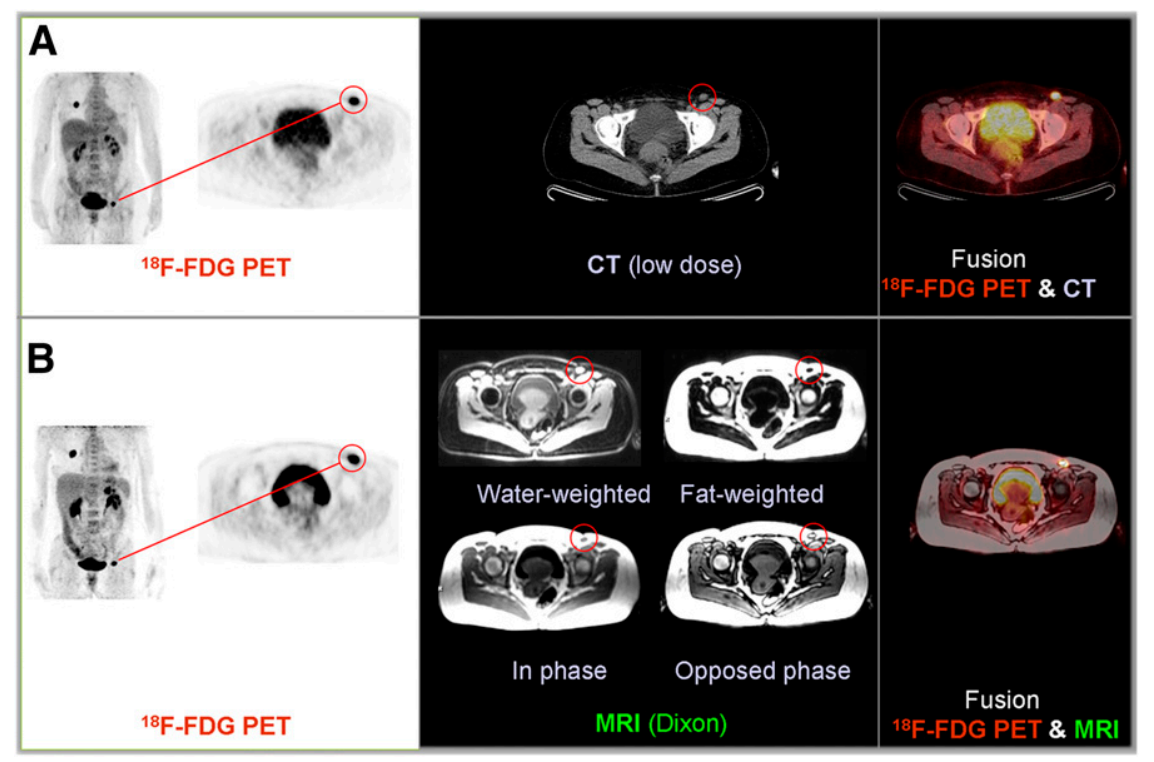

FIGURE 2. Illustration of comparable quality of PET data and feasibility of anatomic allocation by low-dose CT and Dixon MRI in patient with metastases of melanoma: PET/CT (A) and PET/MR (B). (Left) maximum-intensity projection overview of findings with ${ }^{18} \mathrm{~F}-\mathrm{FDG}$ PET. (Middle) Axial slices of both modalities showing inguinal focus, presumably representing lymph node metastasis (labeled with red circles). Structural correlate of PET lesion is detectable on low-dose CT and all 4 Dixon MRI scans. Suggestive lymph node can be identified on all types of images consistently. (Right) Fusion overlay of PET and CT, and fusion overlay of PET and opposed-phase Dixon MRI. 
FIGURE 3. Illustration of comparable quality of PET data and feasibility of anatomic allocation by low-dose CT and Dixon MRI in patient with metastases of melanoma: PET/CT (A) and PET/MR (B). (Left) MIP overview of findings with ${ }^{18} \mathrm{~F}-\mathrm{FDG}$ PET. (Middle) Axial slices of both modalities showing pulmonary focus, presumably representing lung metastasis. Structural correlate of PET lesion is detectable on low-dose CT and on 3 of 4 Dixon MRI scans (labeled with red circles). Pulmonary nodule node cannot be identified on the fat-weighted sequence. (Right) Fusion overlay of PET and CT, and fusion overlay of PET and opposed-phase Dixon MRI.

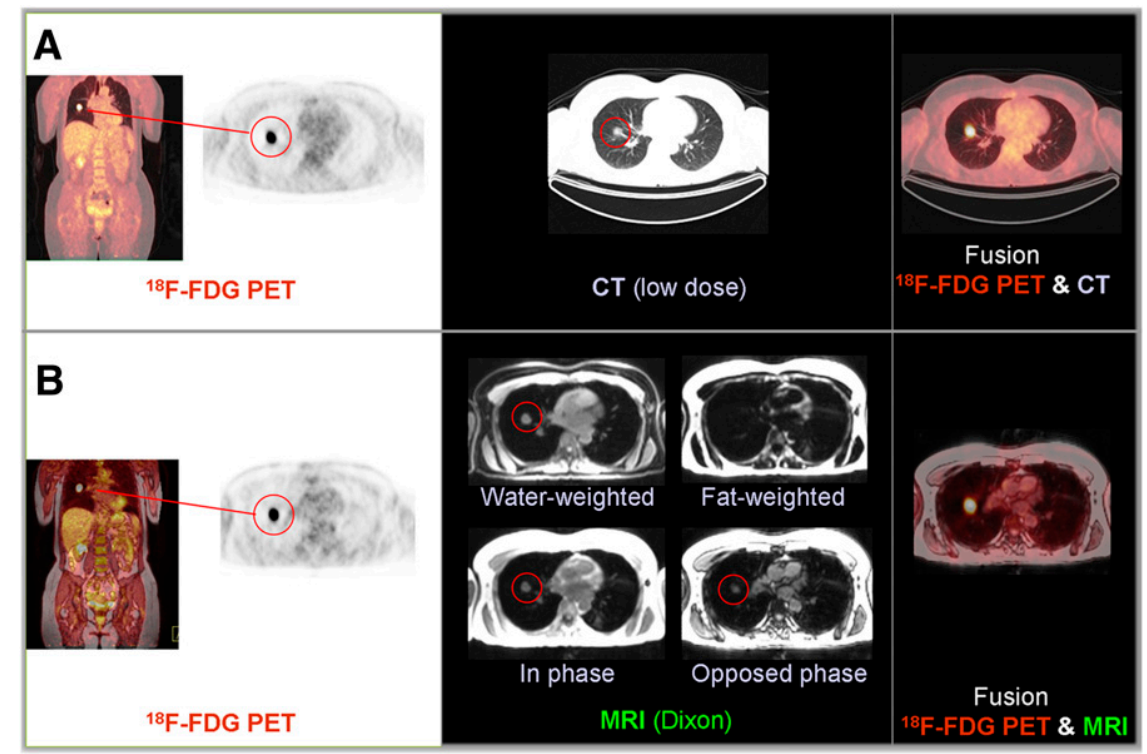

malignant foci) was comparable for both types of instruments. In statistical comparison, neither the number of patients rated positive for suspected lesions nor the total number of detected PET lesions was significantly different between the 2 modalities. In 1 patient, 2 liver lesions observed on PET/CT were missed at first by the team rating the PET/MR data. However, on retrospective analysis, this finding was present also on the PET/MR scan with relatively low lesion-to-background contrast. The PET/MR scan of this patient had been acquired particularly late after injection of the tracer (247 min after injection) for technical reasons. In contrast to other cases, in this case the lesion-tobackground contrast did not increase, because of a strong drop in SUV in both. This patient had a hepatocellular carcinoma, and it has been demonstrated that $k_{4}$ (dephosphorylation kinetic constant) can be elevated in this tumor type, preventing intracellular accumulation of ${ }^{18} \mathrm{~F}-\mathrm{FDG}$ (33). This fact in combination with the late acquisition time and the resulting low count rate may explain the problems in detecting the mentioned liver lesions on the PET/MR scan. We nevertheless decided to include this patient in the study population, as a late acquisition time had not originally been defined as an exclusion criterion.

Regarding subjective rating, the contrast of the lesions was scored comparably high in both imaging modalities. Ratings showed a good correlation between the groups, indicating that lesions rated as being of high contrast on PET/CT were also rated of high contrast on PET/MR. Also, the general image quality was rated to be very good for both modalities, with a somewhat lower rating for PET/MR, most probably because of the later acquisition times of the PET/MR scans. The observers reported partially inhomogeneous liver uptake in some of the PET/MR PET data, but otherwise no systematic artifacts were described.

The PET findings were allocated using the low-dose CT scan for PET/CT and the Dixon MRI sequence for PET/
MR. The Dixon MRI sequence is routinely acquired at every BP for attenuation correction of the PET data in the PET/MR scanner. In a recent study, Eiber et al. suggested that this MRI sequence has a high value for anatomic allocation of PET findings, comparable to low-dose CT (23). Also in our study, MRI-based allocation of the PET findings was possible with good success. Opposed-phase and water-weighted MRI scans were rated best, with no significant difference from low-dose CT-based allocation. The lowest performance was observed for the fat-weighted images. A correlation analysis between the ratings of the quality of anatomic allocation revealed high correlation coefficients between all MRI datasets, indicating that the feasibility of locating lesions was similar with the different available MRI datasets. In contrast, correlation between the scores for all individual MRI scans and the low-dose CT scans was generally low, suggesting that difficulties in anatomic allocation were different in nature for low-dose and MRI-based approaches.

TABLE 4

Mean SUVs

\begin{tabular}{lcrrl}
\hline \multicolumn{1}{c}{ Site } & PET/CT & PET/MR & \multicolumn{1}{c}{$P^{\star}$} & $\rho$ \\
\hline Lesions & $6.1 \pm 3.7$ & $5.4 \pm 3.1$ & $<0.001$ & 0.93 \\
Background & $1.2 \pm 0.9$ & $0.9 \pm 0.6$ & $<0.001$ & 0.92 \\
Lung & $0.36 \pm 0.08$ & $0.29 \pm 0.1$ & 0.002 & 0.5 \\
Liver & $2.4 \pm 0.3$ & $1.6 \pm 0.4$ & $<0.001$ & 0.46 \\
Spleen & $2.0 \pm 0.3$ & $1.7 \pm 0.6$ & 0.025 & $\mathrm{NS}$ \\
Bone & $2.4 \pm 0.4$ & $1.6 \pm 0.5$ & $<0.001$ & 0.65 \\
Muscle & $0.99 \pm 0.19$ & $0.69 \pm 0.16$ & $<0.001$ & $\mathrm{NS}$ \\
\multicolumn{5}{l}{} \\
\end{tabular}




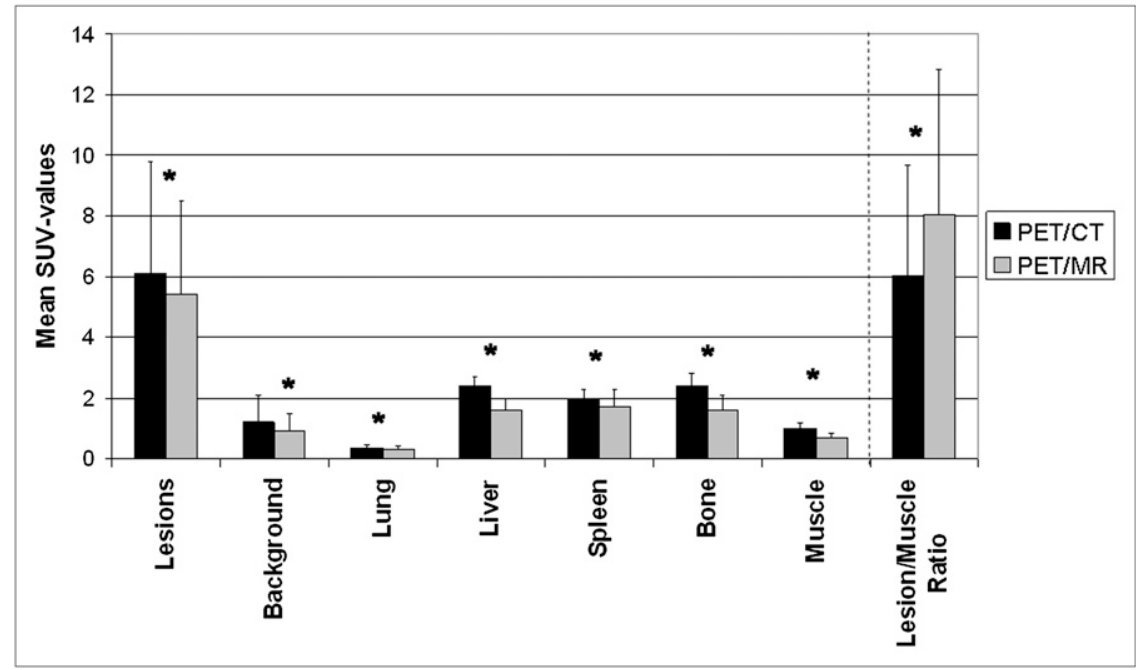

FIGURE 4. Quantitative VOI-based comparison of SUVs between PET/CT and subsequent $P E T / M R$ in suspected lesions and different organ systems in same subjects. Tracer uptake in suspected lesions and in most organ systems shows relative decrease between PET/CT and PET/MR. Lesion-to-muscle ratios show increase between PET/CT and PET/MR due to relatively stronger decrease of background activity over time. Background = all organs combined. *Significant difference.
In contrast to the study of Eiber et al. (23), the best performance regarding locating lesions was observed for opposed-phase and water-weighted MRI scans rather than for in-phase scans. This discrepancy may be due to several basic differences between the 2 studies: First, in contrast to Eiber et al., we analyzed PET data that were from the same patients but were acquired on 2 different PET scanners with different start times after injection and different acquisition times. The proportion of the type of lesions was different between the studies (e.g., 10\% lung lesions in the study of Eiber et al. vs. $19 \%$ in our study). Finally, in contrast to the study of Eiber et al., 2 teams of observers rated exclusively the MRI- or CT-based attenuation-corrected data in our study; that is, raters could not be biased by knowledge of the location of a lesion from a previous rating in another modality. Disregarding these minor differences, we believe that both studies suggest that the MRI Dixon sequence can be used for anatomic allocation of whole-body PET/MR findings in a fashion similar to using low-dose CT for PET/CT. Because the Dixon sequences are acquired anyway for attenuation correction in PET/MR, this option may be valuable for all body regions for which high-quality diagnostic MRI sequences are not acquired because of time restrictions.

Regarding quantitative analysis of tracer uptake by means of SUV-based analysis, significantly lower SUVs have been observed for data acquired on the PET/MR than on the PET/CT scanner for suspected lesions and the different organ systems assessed. The decrease of mean SUV in suspected lesions between PET/CT and PET/MR was much less pronounced $(-9.4 \%)$ than the decrease in organ-to-background ratios (e.g., muscle tissue: -29.3\%). Consequently, the lesion-to-background contrast increased with delayed acquisition time, corresponding to results from previous repeated-imaging studies (34-37). However, whereas a decrease in background SUV over time has been anticipated, higher lesion SUVs would have been expected in the later-acquired PET/MR data, as demonstrated previously (37). This discrepancy may be due to several factors: First, it may be a consequence of systematic differences in the technologic specifications and data-processing algorithms of the 2 scanners. Previous studies have shown that

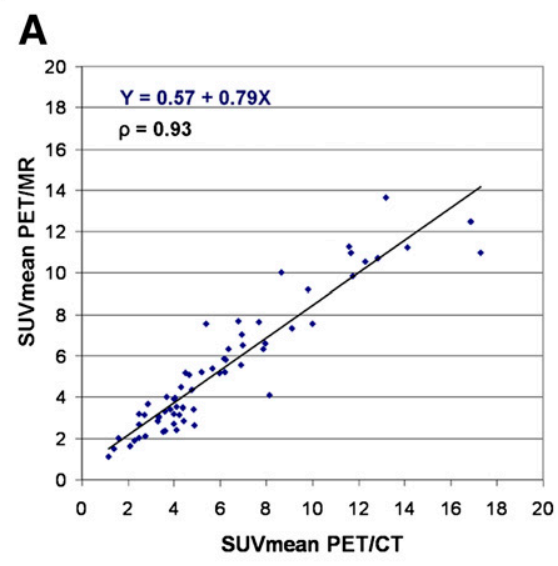

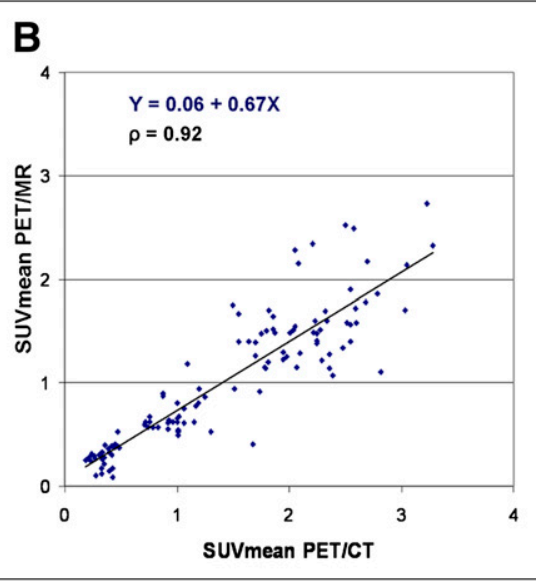

FIGURE 5. Correlation analysis of tracer uptake between PET/CT and subsequent PET/MR in suspected lesions (A) and background (B) (all organs combined), as assessed by SUV-based evaluation. $x$-axis displays quantitative values as obtained by $\mathrm{PET} / \mathrm{CT}$, and $y$-axis displays corresponding values as obtained by PET/MR. High correlation exists between findings from both modalities, despite differences in start time and duration of acquisition. $\rho=$ Spearman correlation coefficient. 
SUV measurements may not be highly reproducible across different scanner types or even between sites using the same scanners $(32,38)$. The obvious differences in SUV reported in these previous studies have been observed despite the fact that phantom or patient data acquired under identical conditions have been used to test the reproducibility of the SUV.

In the current study, distinct differences in scanner technology were present between the 2 modalities, including differences in scanner geometries/fields of view, in scanner beds, in detector technologies, in whether MRI coils are present, and particularly in the approaches used for attenuation correction (MRI-based for PET/MR, CT-based for PET/CT). Several potential effects of these technical issues on the quality of the PET/MR data have been previously discussed (truncation artifacts, ignorance of attenuation by the bone) $(39,40)$. Several approaches for further optimization of data processing have been proposed, particularly with regard to the MRI-based attenuation correction, and are currently under evaluation (40-45). We have not used any of these additional correction algorithms to avoid the potential introduction of further nontransparent variance into the data. Studies of the value of adding these correction algorithms to the processing of the PET/MR data are currently ongoing. In addition to the mentioned technical differences, apparent differences in examination protocols were present in the current study: Longer PET acquisition times per BP were used for PET/MR (4 min) than for PET/CT (2 min), to ensure sufficient quality of the data. The acquisition start of the second scan (PET/MR) was relatively late (140 min after injection), even compared with previous studies on dual time-point examinations (e.g., Kumar et al., 101 min after injection (37)), and was not identical for all subjects. In summary, several different factors may have potentially affected the SUV quantification of the PET/MR data in the current study, and we believe that most probably a combination of factors is responsible for our findings. The current study protocol is not well suited to pinpoint the potential impact of the various technical differences between the scanners on quantification of the PET signal. Thus, future studies should systematically address these issues and test the value of more sophisticated data-processing algorithms, particularly for attenuation correction.

Despite the observed difference in mean SUV, a high correlation $(\rho=0.93)$ was observed between uptake values measured with PET/CT and those measured with PET/MR in suspicious lesions. Furthermore, a high correlation between background SUVs measured with PET/CT and those measured with PET/MR was observed as well. These encouraging results indicate that relative proportions of tracer uptake in lesions and in the background are preserved in PET/MR, as compared with PET/CT, despite different technologies and different approaches for attenuation correction. This implies that the PET/MR scanner is suitable for quantitative evaluation (e.g., of a therapy response) in longitudinal studies but that care should be taken in comparing SUVs between PET/MR and other scanner types. In con- trast to the high correlation across all organ-to-background values, relatively low correlations have been observed between PET/MR and PET/CT SUVs in individual organ systems. The reason for this finding is not clear, but several potential explanations arise: First, the tracer kinetics over time may be subject to stronger interindividual variation in different organ systems (46), and the interval between PET/ $\mathrm{CT}$ and PET/MR was not constant for all patients in the current study. Second, relatively low SUVs have been detected in some organs (e.g., in lungs or muscle), and the respective low counting rates may have affected the reliability of the quantitative evaluation. Third, the fact that most organ values were narrowly grouped around their mean (i.e., showing low variance) may also have hampered the detection of a distinct correlation. Finally, the technical differences between the scanners may also have affected these examinations.

\section{CONCLUSION}

The current study demonstrated that integrated wholebody PET/MR is feasible in a clinical setting and produces images of high quality and without loss of information, as compared with conventional PET/CT. PET data acquired with the new PET/MR technology in patients with oncologic diseases allowed the detection of hypermetabolic lesions suspicious for malignancy with a reliability comparable to that of PET/CT. Despite different attenuation correction approaches (MRI-based for PET/MR, CT-based for PET/ CT), tracer uptake in suspected lesions correlated well between PET/MR and PET/CT. The Dixon MRI sequences acquired for attenuation correction of the PET/MR data were of comparable value to low-dose CT for anatomic allocation of PET findings in the entire body. The acquisition time for PET/MR of the body trunk was short $(<20 \mathrm{~min})$ and can probably be further reduced. In the current study, neither contrast-enhanced diagnostic CT scans nor high-resolution contrast-enhanced diagnostic MRI sequences were performed, and no conclusions on the potential added diagnostic value of PET/MR over PET/CT can be drawn. However, the encouraging findings of the current study may form the necessary foundation for further studies, aiming to prove the added value of PET/MR over conventional PET/CT, when including various diagnostic MRI sequences for specific indications.

\section{DISCLOSURE STATEMENT}

The costs of publication of this article were defrayed in part by the payment of page charges. Therefore, and solely to indicate this fact, this article is hereby marked "advertisement" in accordance with 18 USC section 1734.

\section{ACKNOWLEDGMENTS}

We thank Coletta Kruschke, Sylvia Schachoff, Anna Winter, and the whole PET/MR team for their excellent technical assistance. Furthermore, we thank the cyclotron team for the reliable tracer supply. Finally, we thank all the 
patients who participated in the study. This study was supported by the DFG (Deutsche Forschungsgemeinschaft, Grossgeräteinitiative), which funded the installation of the PET/MR scanner at the Technische Universität München. In addition, this work was supported by grants from the DFG (DR 445/3-1, 4-1) and by the Graduate School of Information Science in Health and the Technische Universität München Graduate School, as well as by the SFB 824. The Department of Nuclear Medicine, Technische Universität München, has established a research cooperation contract with Siemens Healthcare AG, and 3 of the authors have been invited to present lectures on PET/MR by Siemens Healthcare AG. No other potential conflict of interest relevant to this article was reported.

\section{REFERENCES}

1. Antoch G, Bockisch A. Combined PET/MRI: a new dimension in whole-body oncology imaging? Eur J Nucl Med Mol Imaging. 2009;36(suppl 1):S113-S120.

2. Loeffelbein DJ, Souvatzoglou M, Wankerl V, et al. PET-MRI fusion in head-andneck oncology: current status and implications for hybrid PET/MRI. J Oral Maxillofac Surg. 2012;70:473-483.

3. Schlemmer HP, Pichler BJ, Krieg R, Heiss WD. An integrated MR/PET system: prospective applications. Abdom Imaging. 2009;34:668-674.

4. von Schulthess GK, Schlemmer HP. A look ahead: PET/MR versus PET/CT. Eur J Nucl Med Mol Imaging. 2009;36(suppl 1):S3-S9.

5. Zaidi H, Montandon ML, Alavi A. The clinical role of fusion imaging using PET, CT, and MR imaging. Magn Reson Imaging Clin N Am. 2010;18:133-149.

6. Beyer T, Weigert M, Quick HH, et al. MR-based attenuation correction for torsoPET/MR imaging: pitfalls in mapping MR to CT data. Eur J Nucl Med Mol Imaging. 2008;35:1142-1146.

7. Slomka PJ. Software approach to merging molecular with anatomic information. J Nucl Med. 2004;45(suppl 1):36S-45S.

8. Zaidi H, Ojha N, Morich M, et al. Design and performance evaluation of a wholebody Ingenuity TF PET-MRI system. Phys Med Biol. 2011;56:3091-3106.

9. Pichler BJ, Judenhofer MS, Catana C, et al. Performance test of an LSO-APD detector in a 7-T MRI scanner for simultaneous PET/MRI. J Nucl Med. 2006;47:639-647.

10. Ziegler SI, Pichler BJ, Boening G, et al. A prototype high-resolution animal positron tomograph with avalanche photodiode arrays and LSO crystals. Eur J Nucl Med. 2001;28:136-143.

11. Schlemmer HP, Pichler BJ, Schmand M, et al. Simultaneous MR/PET imaging of the human brain: feasibility study. Radiology. 2008;248:1028-1035.

12. Boss A, Bisdas S, Kolb A, et al. Hybrid PET/MRI of intracranial masses: initial experiences and comparison to PET/CT. J Nucl Med. 2010;51:1198-1205.

13. Catana C, Benner T, van der Kouwe A, et al. MRI-assisted PET motion correction for neurologic studies in an integrated MR-PET scanner. J Nucl Med. 2011;52:154-161.

14. Herzog H, Langen KJ, Weirich $\mathrm{C}$, et al. High resolution BrainPET combined with simultaneous MRI. Nuklearmedizin. 2011;50:74-82.

15. Kinahan PE, Hasegawa BH, Beyer T. X-ray-based attenuation correction for positron emission tomography/computed tomography scanners. Semin $\mathrm{Nucl}$ Med. 2003;33:166-179.

16. Hofmann M, Pichler B, Scholkopf B, Beyer T. Towards quantitative PET/MRI: a review of MR-based attenuation correction techniques. Eur J Nucl Med Mol Imaging. 2009;36(suppl 1):S93-S104.

17. Hofmann M, Steinke F, Scheel V, et al. MRI-based attenuation correction for PET/MRI: a novel approach combining pattern recognition and atlas registration. J Nucl Med. 2008;49:1875-1883.

18. Keereman V, Fierens Y, Broux T, De Deene Y, Lonneux M, Vandenberghe S. MRI-based attenuation correction for PET/MRI using ultrashort echo time sequences. J Nucl Med. 2010;51:812-818.

19. Martinez-Möller A, Souvatzoglou M, Delso G, et al. Tissue classification as a potential approach for attenuation correction in whole-body PET/MRI: evaluation with PET/CT data. J Nucl Med. 2009;50:520-526.

20. Zaidi H. Is MR-guided attenuation correction a viable option for dual-modality PET/MR imaging? Radiology. 2007;244:639-642.

21. Schulz V, Torres-Espallardo I, Renisch S, et al. Automatic, three-segment, MRbased attenuation correction for whole-body PET/MR data. Eur J Nucl Med Mol Imaging. 2011;38:138-152.
22. Steinberg J, Jia G, Sammet S, Zhang J, Hall N, Knopp MV. Three-region MRIbased whole-body attenuation correction for automated PET reconstruction. Nucl Med Biol. 2010;37:227-235.

23. Eiber M, Martinez-Moller A, Souvatzoglou M, et al. Value of a Dixon-based MR/PET attenuation correction sequence for the localization and evaluation of PET-positive lesions. Eur J Nucl Med Mol Imaging. 2011;38:1691-1701.

24. Boellaard R, O'Doherty MJ, Weber WA, et al. FDG PET and PET/CT: EANM procedure guidelines for tumour PET imaging: version 1.0. Eur J Nucl Med Mol Imaging. 2010;37:181-200.

25. Elstrom RL, Leonard JP, Coleman M, Brown RK. Combined PET and low-dose, noncontrast CT scanning obviates the need for additional diagnostic contrastenhanced CT scans in patients undergoing staging or restaging for lymphoma. Ann Oncol. 2008;19:1770-1773.

26. Pfannenberg AC, Aschoff P, Brechtel K, et al. Low dose non-enhanced CT versus standard dose contrast-enhanced $\mathrm{CT}$ in combined PET/CT protocols for staging and therapy planning in non-small cell lung cancer. Eur J Nucl Med Mol Imaging. 2007;34:36-44.

27. Jakoby BW, Bercier Y, Watson CC, Bendriem B, Townsend DW. Performance characteristics of a new LSO PET/CT scanner with extended axial field-of-view and PSF reconstruction. IEEE Trans Nucl Sci. 2009;56:633-639.

28. Delso G, Fürst S, Jakoby B, et al. Performance measurements of the Siemens mMR integrated whole-body PET/MR scanner. J Nucl Med. 2011;52:1914-1922.

29. Sommer G, Schaefer AO, Baumann T, Ludwig UA, Fautz HP. Sliding multislice MRI for abdominal staging of patients with pelvic malignancies: a pilot study. $J$ Magn Reson Imaging. 2008;27:666-672.

30. Maki JH, Chenevert TL, Prince MR. The effects of incomplete breath-holding on 3D MR image quality. J Magn Reson Imaging. 1997;7:1132-1139.

31. Delso G, Martinez-Moller A, Bundschuh RA, et al. Evaluation of the attenuation properties of MR equipment for its use in a whole-body PET/MR scanner. Phys Med Biol. 2010;55:4361-4374.

32. Westerterp M, Pruim J, Oyen W, et al. Quantification of FDG PET studies using standardised uptake values in multi-centre trials: effects of image reconstruction, resolution and ROI definition parameters. Eur J Nucl Med Mol Imaging. 2007;34:392-404.

33. Delbeke D, Martin WH. Update of PET and PET/CT for hepatobiliary and pancreatic malignancies. HPB (Oxford). 2005;7:166-179.

34. Conrad GR, Sinha P. Narrow time-window dual-point ${ }^{18} \mathrm{~F}-\mathrm{FDG}$ PET for the diagnosis of thoracic malignancy. Nucl Med Commun. 2003;24:1129-1137.

35. Núñez R, Kalapparambath A, Varela J. Improvement in sensitivity with delayed imaging of pulmonary lesions with FDG-PET. Rev Esp Med Nucl. 2007;26:196207.

36. Thie JA, Hubner KF, Smith GT. Optimizing imaging time for improved performance in oncology PET studies. Mol Imaging Biol. 2002;4:238-244.

37. Kumar R, Loving VA, Chauhan A, Zhuang H, Mitchell S, Alavi A. Potential of dual-time-point imaging to improve breast cancer diagnosis with ${ }^{18} \mathrm{~F}-\mathrm{FDG}$ PET. J Nucl Med. 2005;46:1819-1824.

38. Scheuermann JS, Saffer JR, Karp JS, Levering AM, Siegel BA. Qualification of PET scanners for use in multicenter cancer clinical trials: the American College of Radiology Imaging Network experience. J Nucl Med. 2009;50:1187-1193.

39. Schleyer PJ, Schaeffter T, Marsden PK. The effect of inaccurate bone attenuation coefficient and segmentation on reconstructed PET images. Nucl Med Commun. 2010;31:708-716.

40. Delso G, Martinez-Moller A, Bundschuh RA, Nekolla SG, Ziegler SI. The effect of limited MR field of view in MR/PET attenuation correction. Med Phys. 2010;37:2804-2812.

41. Schöder H, Erdi YE, Chao K, Gonen M, Larson SM, Yeung HW. Clinical implications of different image reconstruction parameters for interpretation of whole-body PET studies in cancer patients. J Nucl Med. 2004;45:559-566.

42. Bal G, Kehren F, Michel C, Watson C, Manthey D, Nuyts J. Clinical evaluation of MLAA for MR-PET [abstract]. J Nucl Med. 2011;52(suppl 1):263.

43. Catana C, van der Kouwe A, Benner T, et al. Toward implementing an MRIbased PET attenuation-correction method for neurologic studies on the MR-PET brain prototype. J Nucl Med. 2010;51:1431-1438.

44. Nuyts J, Michel C, Fenchel M, Bal G, Watson C. Completion of a truncated attenuation image from the attenuated PET emission data. Nuclear Science Symposium Conference Record (NSS/MIC), 2010 IEEE. 2010:2123-2127.

45. Salomon A, Goedicke A, Schweizer B, Aach T, Schulz V. Simultaneous reconstruction of activity and attenuation for PET/MR. IEEE Trans Med Imaging. 2011;30:804-813.

46. Basu S, Kung J, Houseni M, Zhuang H, Tidmarsh GF, Alavi A. Temporal profile of fluorodeoxyglucose uptake in malignant lesions and normal organs over extended time periods in patients with lung carcinoma: implications for its utilization in assessing malignant lesions. Q J Nucl Med Mol Imaging. 2009;53:9-19. 